\title{
Risk Factors for Postsurgical Trichiasis Recurrence in a Trachoma-Endemic Area
}

\author{
Emily S. West, ${ }^{1}$ Harran Mkocha, ${ }^{2}$ Beatriz Munoz, ${ }^{1}$ David Mabey, ${ }^{3}$ Allen Foster, ${ }^{3}$ \\ Robin Bailey, ${ }^{3}$ and Sheila K. West ${ }^{1}$
}

PurPose. The World Health Organization recommends trichiasis surgery to prevent blindness caused by trachoma; however, recurrence is common. Risk factors for recurrence have not been widely studied, particularly in trachoma hyperendemic areas.

Methods. Three hundred ninety-four persons with trichiasis in Tanzania were examined. Participants had undergone surgery $>18$ months before the study. Trichiasis recurrence and active trachoma at study visit were assessed. Ocular swabs were collected and tested for Chlamydia trachomatis. Household members were examined for active trachoma.

Results. Of the surgical eyes, $28 \%$ had recurrence; $40 \%$ of patients had recurrence in one or both eyes. Rates did not vary by time since surgery. Eye-level recurrence rates varied significantly across districts, ranging from $16 \%$ to $38 \%$. Current chlamydial infection among surgical cases was low (6\%) and was not associated with recurrence. Recurrence was associated with tarsal conjunctival inflammation (OR: 2.4; 95\% confidence interval $[\mathrm{CI}]: 1.6-3.8)$ and residence in the Kongwa district (OR: 2.3; 95\% CI: 1.2-4.6)

Conclusions. Recurrence after trichiasis surgery is high, suggesting that vigilant follow-up in surgical cases is needed to reduce blindness. Recurrence is associated with evidence of inflammation in the tarsal conjunctiva, although it is not clear whether the inflammation contributes to recurrence, or is a result of the recurrence. Longitudinal studies of trichiasis patients after surgery are needed. (Invest Ophthalmol Vis Sci. 2005;46:447-453) DOI:10.1167/iovs.04-0600

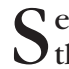
everal countries are developing trachoma control programs that include trichiasis surgery as a main component. The bilamellar tarsal rotation procedure (BTRP) is the surgical strategy endorsed by the World Health Organization (WHO) in cases of trachomatous trichiasis ${ }^{1}$ and is widely used in many parts of Africa. Additional surgical techniques include trabut, cuenod nataf, and tarsal advance and rotation, which have been less well studied. As trachoma control programs are introduced, it is essential that surgical success rates be high and recurrence rates low, to maintain long-term participation.

From the ${ }^{1}$ Dana Center for Preventive Ophthalmology, Johns Hopkins School of Medicine, Baltimore, Maryland; the ${ }^{2}$ Kongwa Trachoma Project, Kongwa, Tanzania; and the ${ }^{3}$ London School of Hygiene and Tropical Medicine, London, United Kingdom.

Supported by the Burroughs Wellcome Fund and the Wellcome Trust and the National Eye Institute (ESW).

Submitted for publication May 26, 2004; revised November 5, 2004; accepted November 11, 2004.

Disclosure: E.S. West, None; H. Mkocha, None; B. Munoz, None; D. Mabey, None; A. Foster, None; R. Bailey, None; S.K. West, None

The publication costs of this article were defrayed in part by page charge payment. This article must therefore be marked "advertisement" in accordance with 18 U.S.C. $\$ 1734$ solely to indicate this fact

Corresponding author: Emily S. West, Dana Center for Preventive Ophthalmology, Johns Hopkins School of Medicine, $600 \mathrm{~N}$. Wolfe Street, Wilmer Room 116, Baltimore, MD 21287; ewest@jhsph.edu.
However, understanding of why surgical patients have recurrence of trichiasis over time is limited. Recent studies have begun to examine long-term recurrence rates, ${ }^{2-4}$ but risk factors have not been fully characterized. In the absence of such knowledge, using data on incident trichiasis, some researchers have begun to suggest that antibiotic treatment for surgical patients and their family members may be an important part of the WHO-endorsed strategy for trachoma control, SAFE. ${ }^{5}$ SAFE denotes Surgery for trichiasis correction, Antibiotics to treat infection, and Face washing and Environmental improvement to improve sanitation. Before such measures are adopted, it is important to determine whether a link exists between trachoma, chlamydial infection, and recurrence of trichiasis. Therefore, we conducted the present study to evaluate the association between trachoma and Cblamydia trachomatis infection in surgical patients and their household members as risk factors for trichiasis recurrence. We also determined longterm rates of trichiasis recurrence in a trachoma-endemic area in central Tanzania.

\section{MeTHODS}

We conducted an observational cohort study of patients who had undergone trichiasis surgery and who lived within five districts (Kongwa, Mpwapwa, Dodoma, Manyoni, and Singida) in central Tanzania. Each of these districts has an established surgery program that maintains up-to-date, complete lists of all trichiasis surgeries performed in the district. These surgical lists serve as the medical records for the district. Surgical cases were identified by using the surgical lists kept by the programs in each district. All men and women who had undergone trichiasis surgery before January 1, 2000, were recruited for participation between June and September 2001.

Participants were asked to provide information on the number of surgeries they had had on each eyelid, to report any use of antibiotics for trachoma treatment within the past year, and to indicate whether they cared for children living within and/or outside the household. Surgical patients were examined for current trachoma status and presence and severity of trichiasis recurrence using WHO's simplified grading scheme. ${ }^{6}$ All individuals living within a surgical participant's household and all children cared for regularly by a surgical participant also were examined for current trachoma status. A household was defined as individuals who use a single entrance into a building that defines their sleeping/cooking area (the area may or may not be subdivided into different rooms). Given this definition, more than one household may reside within the same building.

All participants were tested for the presence of ocular C. trachomatis. For this procedure, a Dacron swab was rubbed across the tarsal plate of the left eye, according to a standard protocol. Care was taken to avoid field contamination. The person who everted the eyelids washed his hands between each ocular examination, and the person who collected the swab never touched the participant during the swabbing procedure, except with the swab tip, which touched the everted lid. Samples were taken of the hands of the person who took the swabs at periodic intervals; all control swabs were negative. Swabs were placed dry in individual vials, kept cold on ice in the field, and then stored at $-20^{\circ} \mathrm{C}$ until they were shipped on ice to the London School of Hygiene and Tropical Medicine (LSHTM) and the Interna- 
tional Chlamydia Laboratory at Johns Hopkins University (JHU) for processing. One-third of the specimens were processed at LSHTM and two-thirds were processed at JHU. Once received, laboratory technicians extracted the DNA from the swabs by adding molecular grade water and vortexing them. A second detergent was then added to finish the preparation of the specimens for analysis. Standard DNA amplification techniques using a $C$. trachomatis qualitative PCR assay (Amplicor) were used to analyze the specimens according to the colorimetric assay in the package insert (Roche Molecular Systems, Branchburg, NJ). Equivocal specimens were reassayed and assigned a positive value if optical densities were still between 0.2 and 0.8 . Ten percent of samples were processed in duplicate at the two laboratories, and no differences were found between the two. Laboratory positivity was the definition for infection with $C$. trachomatis.

The primary outcome measure was trichiasis recurrence, defined as a minimum of one lash touching the globe or evidence of epilation. Evidence of epilation was defined as the presence of visible eyelash stubs. One ophthalmic nurse who had been standardized to an experienced trachoma grader performed all trachoma grading (using the simplified WHO grading scheme) with a $2.5 \times$ magnification loupe and a handheld light. The presence or absence of the five signs was assessed. In particular, because this group of patients would be expected to have scarring, trachomatous inflammation (TI) was graded strictly according to the WHO definition: pronounced inflammatory thickening of the upper tarsal conjunctiva that obscures more than half of the normal deep tarsal vessels because of diffuse inflammatory infiltration or follicles; thickening of the conjunctiva should not be confused with that caused by scarring. ${ }^{1}$ We used a detailed grading scheme to evaluate the severity of trichiasis recurrence. This grading scheme was based on the expanded classification of trichiasis initiated by Reacher et al. ${ }^{7}$ For this classification, the ophthalmic nurse counted and recorded the number of lashes touching any part of the globe and the number touching the cornea. The location was determined by dividing the eyelid into thirds. Lashes touching the globe were recorded based on the location from which they grew: nasally, centrally, or temporally. Given the nature of the study, the clinical observer was not masked to the primary outcome when assessing risk factors such as district, presence of children in the household, or clinical trachoma grade. However, the assessment of recurrence in the cases preceded assessment of family members for trachoma, and all trachoma assessment was completed before the laboratory findings were made known.

With the exception of pregnant women, participants with signs of active trachoma (follicular trachoma [TF]/trachomatous inflammationintense [TI]), were treated with azithromycin, in accordance with the current standard of treatment under the Tanzania National Trachoma Control Program. Pregnant women were given a tube of tetracycline and instructed on twice daily administration for 6 weeks. All procedures were approved by the Johns Hopkins Joint Committee on Clinical Investigation (JCCI) and the National Medical Research Institute of Tanzania (NMRI), in accordance with the Declaration of Helsinki.

All questionnaires were double-data entered by two separate individuals with a customized database (MS Access; Microsoft, Redmond, WA). Discrepancies were reconciled with original data forms. Cross tabulations were created to characterize the population and to evaluate unadjusted associations between potential risk factors and trichiasis recurrence by using likelihood ratio $\chi^{2}$ tests. These analyses were performed primarily at the person level for initial evaluation of the study population. Multivariate analyses were conducted at the eye level. Individuals who had bilateral surgery contributed both eyes to the analyses. To account for the correlation between eyes, logistic regressions with generalized estimating equations ${ }^{3}$ were used to evaluate the association between each risk factor and trichiasis recurrence, after adjusting for other covariates in the model. These analyses were conducted using the GENMOD procedure (SAS ver. 8.0; SAS, Cary, NC). Exchangeable correlation was assumed in these logistic modelsthat is, correlation between pairs was assumed to be the same within pairs.
Additional models were built to examine the role of trachoma among family members and trichiasis recurrence. Trachoma (or infection) in the surgical patient correlated highly with trachoma (or infection) in household members, and we hypothesized both were related to trichiasis recurrence. Therefore, the two factors were examined separately. Infection among household members was evaluated in three ways. For each method, variables were created to indicate whether no person, one person, or more than one person was infected. The three methods included the following: all household members (including adults but not including daycare children who did not sleep in the house); children aged $<9$ years living in the household; and all children aged $<9$ years cared for by the surgical patient, either living in the household or children in daycare who did not sleep in the house.

\section{Results}

\section{Recruited Participants}

Of the potential 601 surgical participants identified, $394 \mathrm{pa}-$ tients were alive, located, and consented to participate; 56 had died; 148 had moved out of the district or could not be located; and 3 refused to participate (Fig. 1). Seven hundred fifty-three household members and 124 children cared for by surgical patients also agreed to participate. Twelve household members refused to participate, and 17 household members could not be located. Surgical participants were similar to nonparticipants except that nonparticipants were more likely to have had surgery 4 to 5 years before the study than participants (Table 1). There were no differences by age, gender, or district between participants and nonparticipants. Ten participants who were otherwise eligible for the study reported multiple trichiasis surgeries in each eye. These individuals were excluded from all remaining analyses, because recurrence is already known to be highly associated with prior surgery. Study results are presented for the remaining 384 participants in whom at least one eye underwent a single trichiasis surgery. Among the 384 participants, personal and familial characteristics varied across district of residence (Table 2). Overall, 129 surgical participants had unilateral surgery, and 255 had bilateral surgery. Seven of the 255 patients who had bilateral surgery reported multiple surgeries in one eye. For these seven individuals, only the one eye with no prior surgeries was included in the analyses. In summary, 630 eyes of 384 patients were available for analyses (Table 3 ).

\section{Recurrence Rates}

Overall, $27.9 \%$ of surgical eyes had trichiasis recurrence; of these, $41.1 \%$ of bilateral cases had recurrence in at least one eye, and $11.7 \%$ had recurrence in both eyes. Of individuals who had unilateral surgery $50.4 \%$ were found to have nonsurgical trichiasis in the fellow eye (Table 3), and $31.8 \%$ of unilateral surgeries had failed by the time of follow-up. Trichiasis recurrence rates varied significantly by district $(P=0.0001)$ with Kongwa having the highest rate and Singida having the lowest rate (Table 4). We found that beyond 18 months, time since surgery did not appear to be associated with trichiasis recurrence and that the recurrence rate was already high by that time point (Fig. 2).

\section{TI and Infection}

Evidence of TI was found at the time of follow-up in $44.8 \%$ of eyes (range, $20.5 \%$ in Singida to $67.5 \%$ in Kongwa), whereas infection with C. trachomatis was found in 6.3\% (range, $0 \%$ in Singida to $12.0 \%$ in Kongwa). Among the four districts with villages that were part of the National Trachoma Control pro- 
FigURE 1. Summary of study participants. "Two eyes became phthisical after surgery and were not evaluated for trichiasis, and seven eyes had multiple surgeries and were excluded from the analyses.

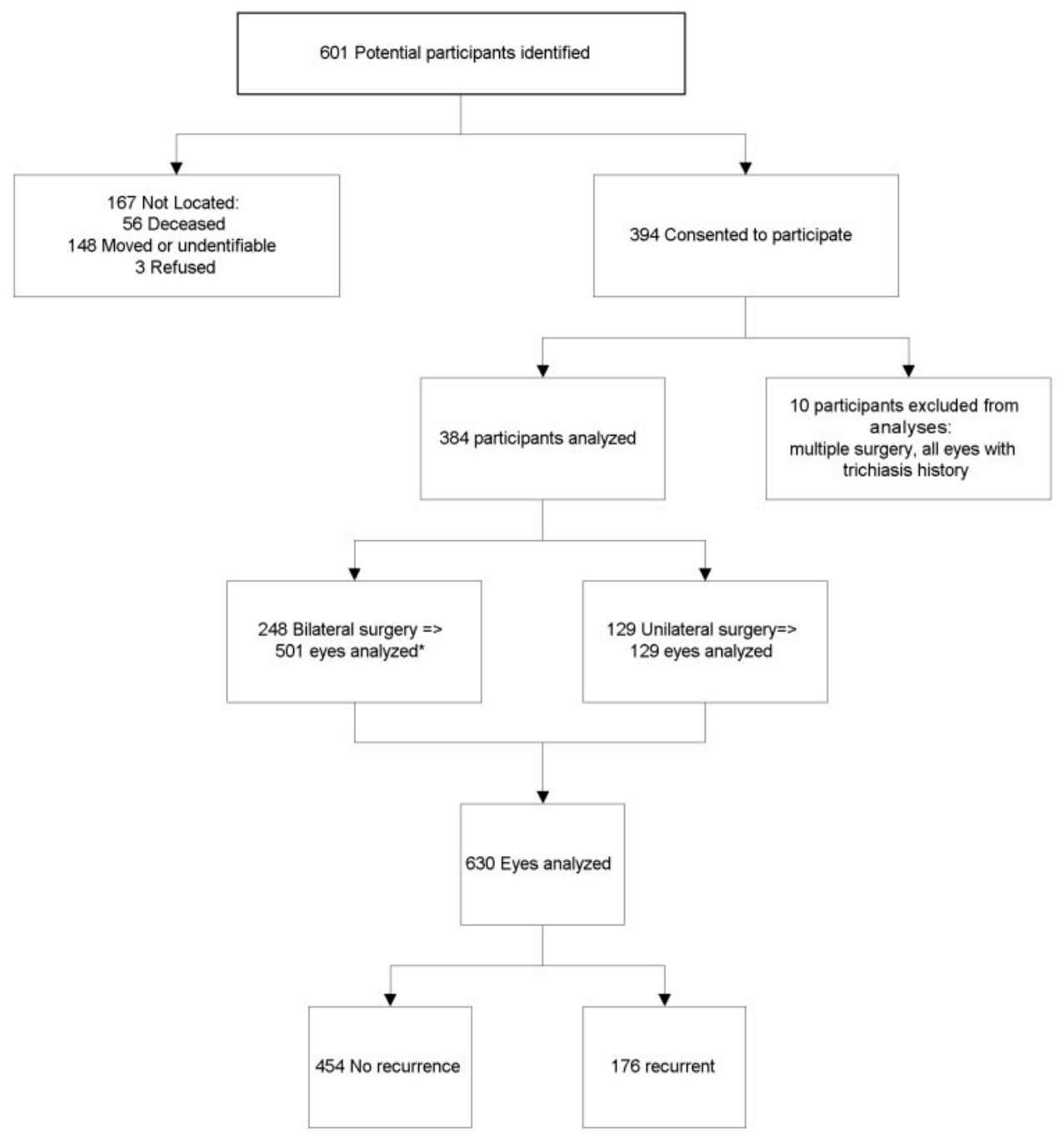

gram (Singida was not at the time), the percentage of individuals reporting azithromycin use during the past year ranged from $22 \%$ to $50 \%$. Infection rates among household members varied significantly across districts. In Manyoni and Singida,

TABLE 1. Characteristics of Participating Surgical Cases Compared to Non-Participants

\begin{tabular}{lccc}
\hline \multicolumn{1}{c}{ Characteristic } & Participants & Nonparticipants & $\boldsymbol{P}^{*}$ \\
\hline Individuals $(n)$ & 394 & 167 & \\
Age (\%) & & & \\
$\quad<45$ & 9.3 & 12.7 & 0.07 \\
$\quad 45-64$ & 33.7 & 41.6 & \\
$\quad$ 65 & 57.0 & 45.8 & \\
Female (\%) & 81.7 & 80.5 & 0.7 \\
Years since surgery (\%) & 46.3 & 43.1 & 0.01 \\
$\quad$ 3 & 29.8 & 38.9 & \\
$4-5$ & 19.5 & 18.0 & \\
6-7 & 4.5 & 0.0 & \\
$\geq 8$ & & & \\
Live in district (\%) & 29.2 & 24.0 & 0.2 \\
$\quad$ Kongwa & 20.3 & 15.6 & \\
Mpwapwa & 16.8 & 17.4 & \\
Dodoma & 15.0 & 17.4 & \\
Manyoni & 18.8 & 25.8 & \\
Singida & & & \\
\hline
\end{tabular}

${ }^{*}$ Likelihood ratio $\chi^{2}$ test.
$>98 \%$ of households had no one infected, whereas in the Kongwa, Dodoma and Mpwapwa districts $>19 \%$ of households had at least one infected household member. In addition, in Kongwa and Mpwapwa, 10 surgical patients cared for infected children who did not live in their households.

\section{Risk Factors}

Statistically significant associations between trichiasis recurrence and TI in the surgical eye and having two or more household members with TI (Table 5) were found. After adjustment for gender and district, individuals aged 45 to 64 years seemed more likely to have recurrence than individuals aged $<45$ years (OR: $2.0 ; 95 \%$ confidence interval [CI]: $0.9-4.5$ ).

Multivariate analyses demonstrated a significant association between the presence of recurrent trichiasis and eyes with TI (OR: 2.4; CI: 1.6-3.8) and residence in Kongwa (OR: 2.3; CI: 1.2-4.6; Table 6). In addition, increased age was associated with recurrence. Infection with $C$. trachomatis in surgical patients was not significantly related to recurrence, even after removal of individuals who reported taking azithromycin within the past year. Multivariate models of infection among household members in families in which surgical patients did not report azithromycin use suggested that having two or more infected children living in the household was associated with increased risk of trichiasis recurrence (OR: 5.9; CI: 1.9-18.9). These results, however, are based on small numbers; there are 
TABLE 2. Characteristics of 384 Surgical Cases and Their Households

\begin{tabular}{|c|c|c|c|c|c|c|c|}
\hline & Kongwa & Mpwapwa & Dodoma & Manyoni & Singida & Total & $\boldsymbol{P}^{*}$ \\
\hline Surgical cases $(n)$ & 115 & 80 & 66 & 59 & 74 & 384 & \\
\hline \multicolumn{8}{|l|}{ Personal characteristics (\%) } \\
\hline \multicolumn{8}{|l|}{ Age } \\
\hline$<45$ & 13.9 & 12.5 & 9.4 & 5.1 & 2.7 & 9.3 & 0.005 \\
\hline $45-64$ & 35.2 & 43.1 & 34.4 & 30.5 & 23.3 & 33.5 & \\
\hline $65+$ & 50.9 & 44.4 & 56.3 & 64.4 & 74.0 & 57.2 & \\
\hline Female & 79.8 & 80.5 & 87.7 & 79.7 & 83.8 & 82.0 & 0.5 \\
\hline Bilateral surgery & 7.8 & 53.3 & 93.9 & 74.6 & 62.2 & 66.4 & $<0.001$ \\
\hline Infected with $C$. trachomatis & 12.0 & 7.8 & 4.7 & 3.4 & 0.0 & 6.3 & 0.001 \\
\hline Reported azithromycin use in past year & 22.0 & 50.7 & 26.2 & 23.7 & 0.0 & 24.5 & $<0.001$ \\
\hline Surgical eyes with TI/inflammation $\dagger$ & 67.5 & 62.2 & 35.4 & 24.7 & 20.5 & 44.8 & $<0.001$ \\
\hline \multicolumn{8}{|l|}{ Family characteristics (\%) } \\
\hline \multicolumn{8}{|l|}{ Household size $\neq$} \\
\hline 1 Person & 7.3 & 13.0 & 21.5 & 39.0 & 29.7 & 20.1 & $<0.001$ \\
\hline 2-5 People & 74.3 & 75.3 & 64.6 & 57.3 & 68.9 & 69.5 & \\
\hline$\geq 6$ People & 18.4 & 11.7 & 13.9 & 1.7 & 1.4 & 10.4 & \\
\hline Median household size $\ddagger$ & 3.0 & 3.0 & 3.0 & 2.0 & 2.0 & 3.0 & $<0.001$ \\
\hline Households with $\geq 1$ children age $<9$ years & 46.8 & 44.2 & 43.1 & 27.1 & 29.7 & 39.3 & 0.04 \\
\hline \multicolumn{8}{|l|}{ Infected household members: } \\
\hline None & 82.1 & 78.4 & 81.3 & 98.2 & 98.6 & 86.7 & $<0.001$ \\
\hline 1 Person & 15.1 & 12.2 & 12.5 & 1.8 & 1.4 & 9.5 & \\
\hline $2+$ Persons & 2.8 & 9.5 & 6.3 & 0.0 & 0.0 & 3.8 & \\
\hline Caring for $\geq 1$ outside children & 22.9 & 9.1 & 7.7 & 6.8 & 18.9 & 14.3 & 0.006 \\
\hline \multicolumn{8}{|l|}{ Caring for: } \\
\hline No infected children & 92.7 & 97.4 & 100.0 & 100.0 & 100.0 & 97.4 & $0.04^{\S}$ \\
\hline 1 Infected child & 5.5 & 2.6 & 0.0 & 0.0 & 0.0 & 2.1 & \\
\hline$\geq 2$ Infected children & 1.8 & 0.0 & 0.0 & 0.0 & 0.0 & 0.5 & \\
\hline
\end{tabular}

${ }^{*}$ Likelihood ratio $\chi^{2}$ test unless otherwise noted.

$\dagger N=630$ eyes.

‡ Includes surgical case.

${ }^{s}$ Fisher's exact test.

8 of 276 observations in which two or more infected children live in the household.

\section{Discussion}

Within this trachoma-endemic area of central Tanzania, recurrence of trichiasis after surgery is a serious problem. The high rate of recurrence emphasizes the need for a community-based intensive surgical follow-up program with scheduled visits after surgery. At the time of this study (at least 18 months after surgery), $50 \%$ of individuals had trichiasis in at least one eye (either surgically treated or not) and $28 \%$ had trichiasis in both eyes, highlighting the significant risk of potential blindness among surgical patients. This finding is higher than in initial studies that suggested recurrence rates near $20 \% 2$ years after surgery $^{7,10}$ but in line with recent studies that have found recurrence rates ranging from $16 \%$ to $60 \% .^{2-4}$

We did not find an association between chlamydial infection and recurrence. Previous studies have shown infection rates of $15 \%$ among women with scarring, ${ }^{11}$ compared with our finding of $6.3 \%$. There are several possible explanations for this finding. We collected ocular specimens from patients who

TABLE 3. Trichiasis Recurrence among 384 Surgical Cases According to Number of Surgical Eyes

\begin{tabular}{|c|c|c|c|c|}
\hline & \multicolumn{3}{|c|}{ Surgery } & \multirow[b]{2}{*}{ Total } \\
\hline & Bilateral & Unilateral & $\begin{array}{c}\text { Bilateral } \\
\text { Study Eye } 1 \text { Surgery, } \\
\text { Contralateral Eye } \\
\text { Multiple Surgeries }^{\mathbb{S}}\end{array}$ & \\
\hline Individuals examined $(n)$ & 248 & 129 & 7 & 384 \\
\hline \multicolumn{5}{|l|}{ Recurrence (\%)* } \\
\hline Both eyes & 11.7 & NA & NA & \\
\hline One eye & 29.4 & 31.8 & 85.7 & \\
\hline None & 58.9 & 68.2 & 13.3 & \\
\hline Trichiasis present $\dagger$, fellow eye (\%) & NA & 50.4 & NA & \\
\hline Eyes included in analyses $(n)$ & $494 \ddagger$ & 129 & & 630 \\
\hline
\end{tabular}

* Recurrence defined as having $\geq 1$ lashes touching the globe and/or evidence of recent epilation at time of study visit.

† Trichiasis present at time of study visit. Eye had not had trichiasis surgery.

₹ Two eyes that had had trichiasis surgery were phthisical by the time of the study visit and could not be examined.

${ }^{\S}$ Eyes with multiple surgeries excluded from table. 
TABle 4. Percentage of 630 Eyes with Trichiasis Recurrence by District

\begin{tabular}{lcccc}
\hline \multicolumn{1}{c}{ District } & $\begin{array}{c}\text { Eyes Analyzed } \\
(\boldsymbol{n})\end{array}$ & $\begin{array}{c}\text { Recurrence } \\
(\%)\end{array}$ & OR & 95\% CI \\
\hline Singida & 168 & 16.0 & Reference & - \\
Manyoni & 116 & 19.4 & 1.3 & $0.6-2.8$ \\
Dodoma & 124 & 28.2 & 2.0 & $1.0-4.0$ \\
Mpwapwa & 103 & 32.8 & 2.6 & $1.3-5.0$ \\
Kongwa & 119 & 38.1 & 3.2 & $1.7-5.9$ \\
Total & 630 & 27.9 & & \\
\hline
\end{tabular}

had undergone trichiasis surgery, many of whom had severely scarred lids. These lids have much less conjunctival epithelial tissue than do normal, healthy lids, making specimen collection difficult. In addition, azithromycin has been used in four of the five districts, which may have a long-term impact on the prevalence of chlamydial infection in the community. Our data do not reflect infection before recurrence, but rather a crosssectional association. However, mass treatment programs had been newly implemented in some areas of this region within one year before this study, and recurrence certainly could have occurred before distribution of mass treatment. Thus, it is difficult to interpret the role of treatment in this cohort. In this study, recurrence of trichiasis was found to be associated with TI in the surgical eye. Grading TI in the presence of scarring always raises concerns, as scarring also can obscure tarsal vessels, leading to an overcall of TI. However, we were careful to differentiate obscuration of vessels due to scarring from that due to inflammation, in accordance with the WHO criteria. The question of whether the inflammation was due to chlamydial infection is another issue. Though the prevalence of infection in patients was low, the results showed an association between chlamydial infection and TI, after adjustment for age and gender. Overall, $10 \%$ of eyes with inflammation tested positive for chlamydia compared with $2 \%$ of eyes without inflammation $(P<0.001)$, suggesting that at least some of the inflammation was due to trachoma. However, we cannot rule out the possibility that some of the inflammation was due to the recurrence itself causing irritation or to the presence of other infections. Only longitudinal studies can directly answer the question of the temporality of the association.

Recurrence of trichiasis after surgery may be due to factors associated with the technique, skill, and experience of the surgeon and/or factors related to the disease process and patients response. It is likely that surgical factors cause early recurrence, whereas ongoing disease factors will contribute more significantly to late recurrence. We found a high rate of recurrence as early as 18 months after surgery. We could not specifically evaluate the surgeons' skill and technique in this study. The only surrogate, albeit an imperfect one, is district, and we did find differences in district-specific recurrence rates, discussed in more detail later. All multivariate analyses included districts as covariates to help control for the differences across districts, including surgeons.

Trichiasis recurrence also was associated with residence in the Kongwa district. In Tanzania, one or two individuals perform nearly all trachomatous trichiasis (TT) surgeries for a specific district, with the exception of Kongwa, where trichiasis training is performed regularly involving many trainee surgeons. Thus, differences in surgical experience may be a factor. This possibility should be examined further in future research. Another possible explanation for the association with the Kongwa district is that after adjustment for other risk factors, both Kongwa and Mpwapwa had significantly higher rates of recurrence than did the remaining districts. These districts also had higher rates of infection and active disease among household members; thus, district may be a marker for overall infection in the community, which could be a contributing factor to recurrence. Negrel and Khandekar also found that individuals living in high-risk areas had significantly higher rates of recurrence (OR: 3.9 and 2.3 , respectively). ${ }^{3,4}$ Within districts where TI was prevalent, surgical patients living with other individuals who had TI were more likely to have trichiasis recurrence than were those who lived alone or with other household members who did not have TI. These findings suggest an association between trichiasis recurrence and exposure to trachoma in household members.

Models examining recurrence as a function of infection among household members suggested an association between infection in children and recurrence. In these models, the more exposure a surgical patient had to infected children, the more likely the patient was to have recurrence. The risk of recurrence increased when two or more children were infected. However, these models are based on a limited number of households in which two or more children were infected $(n=8)$ and thus, even though the incidence was significant,

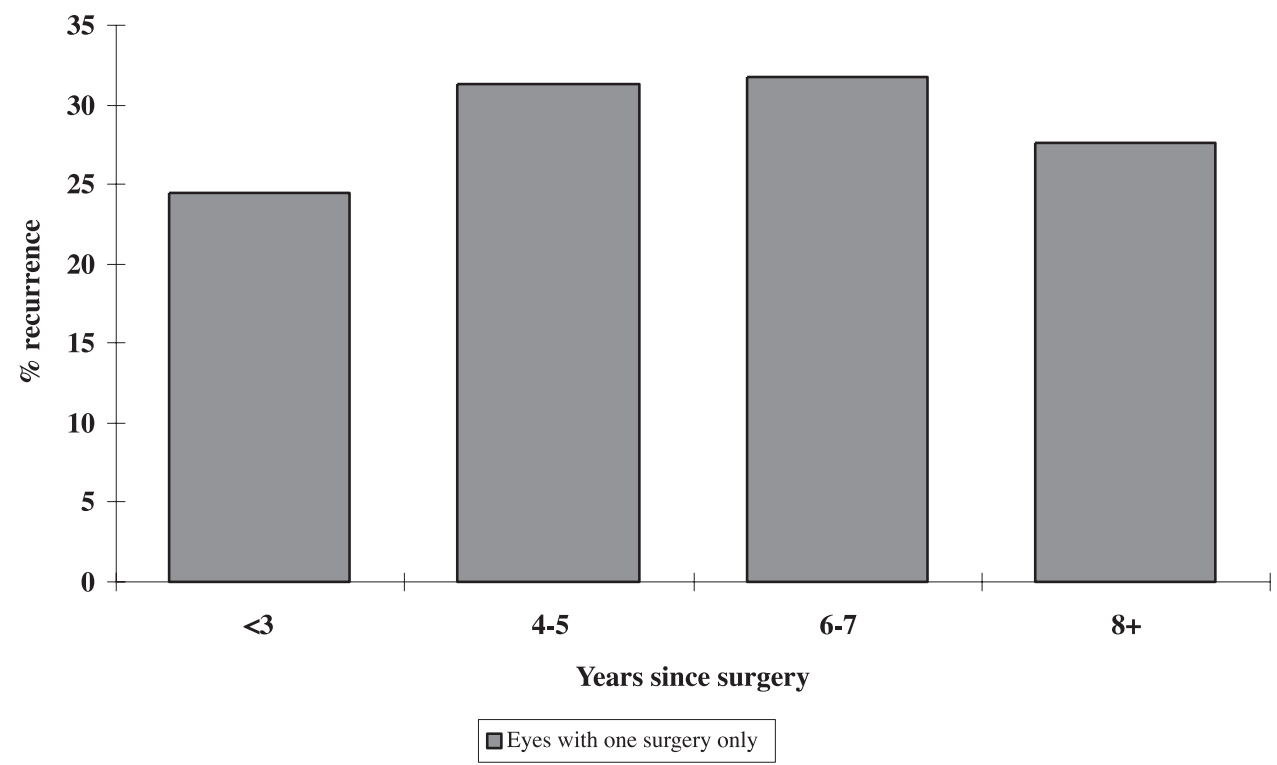

Figure 2. Percentage of eyes with recurrence with trichiasis as a function of years since surgery. 
TABLE 5. Total Number and Percentage of Surgical Cases with Trichiasis Recurrence According to Presence or Absence of Potential Risk Factors*

\begin{tabular}{|c|c|c|c|c|}
\hline Risk Factor & $N$ & $\begin{array}{l}\text { Recurrence } \\
(\%)^{*}\end{array}$ & $\begin{array}{l}\text { Unadjusted } \\
\text { OR (CI) }\end{array}$ & $\begin{array}{c}\text { Multiple } \\
\text { Adjusted OR } \\
\text { (CI) }\end{array}$ \\
\hline \multicolumn{5}{|l|}{ Age category } \\
\hline$<45$ & $11 / 35$ & 31.4 & Reference & Reference \\
\hline $45-64$ & $55 / 126$ & 43.7 & $1.7(0.8-3.7)$ & $2.0(0.9-4.5)$ \\
\hline$\geq 65$ & $80 / 215$ & 37.2 & $1.3(0.6-2.8)$ & $1.7(0.8-3.8) \dagger$ \\
\hline \multicolumn{5}{|l|}{ Gender } \\
\hline Male & $24 / 63$ & 34.8 & Reference & Reference \\
\hline Female & $126 / 315$ & 40.0 & $1.3(0.7-2.2)$ & $1.3(0.7-2.2) \ddagger$ \\
\hline \multicolumn{5}{|c|}{ Infection with C. trachomatis } \\
\hline No & $138 / 358$ & 38.6 & Reference & Reference \\
\hline Yes & $10 / 24$ & 41.7 & $1.1(0.5-2.6)$ & $0.7(0.3-1.7)^{\S}$ \\
\hline \multicolumn{5}{|l|}{ Inflammation/TI" } \\
\hline No & $57 / 315$ & 18.0 & Reference & Reference \\
\hline Yes & $93 / 244$ & 38.1 & $2.8(1.9-4.3)$ & $2.4(1.6-3.8)^{\|}$ \\
\hline Data missing & $26 / 71$ & 36.6 & NA & NA \\
\hline \multicolumn{5}{|c|}{$\begin{array}{l}\text { Any trachoma (TF and/or TI) in } \\
\text { household }\end{array}$} \\
\hline No & $101 / 277$ & 36.5 & Reference & reference \\
\hline Yes & $49 / 107$ & 45.8 & $1.5(0.9-2.3)$ & $1.3(0.8-2.1) \dagger$ \\
\hline \multicolumn{5}{|c|}{$\begin{array}{c}\text { Inflammatory trachoma in } \\
\text { household }(\mathrm{TI} \pm \mathrm{TF})\end{array}$} \\
\hline No & $124 / 333$ & 37.2 & Reference & Reference \\
\hline 1 Person & $17 / 38$ & 44.7 & $1.4(0.7-2.7)$ & $1.1(0.5-2.3)^{\S}$ \\
\hline$\geq 2$ People & $9 / 13$ & 69.2 & $3.8(1.1-12.6)$ & $3.7(1.0-13.3)^{\S}$ \\
\hline
\end{tabular}

* Recurrence in at least one eye.

$\dagger$ Adjusted for district and gender.

$\ddagger$ Adjusted for district and age

${ }^{\Im}$ Adjusted for district, age and gender.

${ }^{\|} N=630$ eyes

ๆ 71 eyes missing inflammation status because they could not be everted.

may not explain a large number of the recurrences and should be interpreted with caution.

It is logical that exposure to children would contribute to the risk of recurrence in these households. The Azithromycin in Control of Trachoma (ACT) trial conducted in Tanzania showed that $C$. trachomatis infection is more common among children. ${ }^{12}$ More recently, it has been shown that heavy infections, determined by quantitative PCR, are also more common in this age group. ${ }^{13}$ Congdon et al. ${ }^{14}$ demonstrated that women who cared for children with active trachoma were three times more likely to have active trachoma than women who did not

TABLe 6. Multivariate Logistic Model Evaluating the Association between Personal Characteristics and Trichiasis Recurrence among 630 Postsurgical Eyes*

\begin{tabular}{ll} 
Variable & $\begin{array}{c}\text { Odds Ratio } \\
(\mathbf{9 5 \%} \mathbf{C I})\end{array}$ \\
\hline Age $(\mathrm{y})$ & \\
$<45$ & Reference \\
$45-64$ & $2.3(1.0-4.9)$ \\
$65+$ & $2.1(1.0-4.5)$ \\
Female & $1.2(0.7-2.1)$ \\
Inflammation/TI in surgical case & $2.4(1.6-3.8)$ \\
District & \\
Kongwa & $2.3(1.2-4.6)$ \\
Mpwapwa & $1.5(0.7-3.2)$ \\
Dodoma & $1.9(0.9-4.0)$ \\
Manyoni & $1.1(0.5-2.6)$ \\
Singida & Reference \\
\hline
\end{tabular}

* Generalized estimating equations methods used to account for correlation between eyes. care for children. The belief behind this association is that caregivers are more likely to be exposed to infection than noncaregivers because of the frequent, close contact that they have with children. However to date, no association between simply caring for children and incident trichiasis has been demonstrated. ${ }^{15}$ In the present study, we did not find an association between simply caring for children and trichiasis recurrence. However, when the analyses were confined to the risk associated with infection in children, a substantially increased risk of recurrence was observed. Further longitudinal studies of risk of recurrence according to infection in households, especially in children, are warranted.

We limited analyses of infection to observations in which the surgical case reported no azithromycin use during the previous year because individuals who took the antibiotic are much less likely to have infection. ${ }^{12}$ We used reported use of azithromycin among surgical patients as a proxy for households who took advantage of azithromycin distribution during the national distribution program. Including azithromycin users in the model did not change the interpretation of results, but it weakened the association between infection in the household and recurrence. This result is not surprising, because ongoing transmission within the families that might have contributed to the recurrence would have been disrupted by recent azithromycin use. Given the cross-sectional nature of the data collection for this study, it is not possible to determine the temporal association between infection or inflammation and trichiasis recurrence. However, data have shown that trachoma clusters in families, and across time, the trachoma level within households is fairly constant. ${ }^{16-18}$ Thus, it is likely that individuals who had infection at the time of this study have had repeated bouts of infection since the time that the surgical 
patient had trichiasis surgery. The current bout of infection most likely occurred after the surgical patient had a recurrence but probably represents infections that the person had been exposed to over time.

We were not able to evaluate the severity of the scarring, or the severity of the trichiasis, before surgery. Severity of trichiasis before surgery does predict recurrence. ${ }^{7,19,20}$ It is possible that some of the differences by district reflect differences in the levels of severity in persons who presented for surgery, but baseline severity in this study is unknown. Previous studies have shown that persistent TI in children is associated with incident scarring, ${ }^{21}$ suggesting that ongoing inflammation may drive the scarring process. Thus, our finding of inflammation associated with recurrence may reflect both the severity of scarring before surgery and an ongoing scarring process as factors in the recurrence of trichiasis after surgery. Further longitudinal studies of trichiasis patients after surgery would enable more precise determination of the contribution of these elements.

In summary, these data suggest that inflammation, in part due to inflammatory trachoma, is associated with trichiasis recurrence and that trachoma within households of surgical patients is a risk factor and may be an important source of infection in surgical patients. Prospective studies of these factors in surgical patients are needed. Given the high rates of recurrence in this area, vigilant follow-up of patients and review of surgical practices are indicated.

In accordance with WHO guidelines promoting SAFE for trachoma control, several countries are in the process of developing national programs with emphasis on trichiasis surgery. As these programs are introduced, it is essential that surgical results be promising. It is important to move forward in determining reasons for high recurrence rates and to work toward reducing risk factors for recurrence.

\section{Acknowledgments}

The authors thank those in the Kongwa Trachoma Project, specifically Frank Migoi, Julieth Mihmili, and Andrew Kayengoya, and Jonathan Miller, MD (Johns Hopkins University), for invaluable assistance in data collection and Aura Aguirre, Angels Natividad, Charlotte Gaydos, and Patti Rizzo for assistance with specimen processing.

\section{References}

1. World Health Organization. Primary Health Care Level Management of Trachoma. New York: World Health Organization Programme for the Prevention of Blindness; 1993.

2. Bowman R, Jatta B, Faal H, Bailey R, Foster A, Johnson GJ. Longterm follow-up of lid surgery for trichiasis in the Gambia: surgical success and patient perceptions. Eye. 2000;14:864-868.
3. Negrel AD, Chami-Khazraji Y, Arrache M, Ottmani S, Mahjour J. The quality of trichiasis surgery in the kingdom of Morocco (in French). Sante. 2000;10:81-92.

4. Khandekar R. Recurrence of trichiasis: a long-term follow up study in the Sultanate of Oman. Opbthalmic Epidemiol. 2001;8:155161.

5. Bowman R, Faal H, Myatt M, et al. Longitudinal study of trachomatous trichiasis in the Gambia. $\mathrm{Br} \mathrm{J} \mathrm{Opbthalmol.} \mathrm{2002;86:}$ $339-343$.

6. Thylefors B, Dawson R, Jones BR, et al. A simple system for the assessment of trachoma and its complications. Bull World Health Organ. 1987;65:477-483.

7. Reacher MH, Munoz B, Alghassany A, Daar AS, Elbualy M, Taylor HR. A controlled trial of surgery for trachomatous trichiasis of the upper lid. Arch Opbthalmol. 1992;110:667-674.

8. Diggle P, Liang K, Zeger S. Analysis of Longitudinal Data. Oxford, UK: University Press; 1994.

9. Liang $\mathrm{K}$, Zeger S. Longitudinal data analysis using generalized linear models. Biometrika. 1986;73:13-22.

10. Bog H, Yorston D, Foster A. Results of community-based eyelid surgery for trichiasis due to trachoma. Br J Opbthalmol. 1993;77: 81-83.

11. Munoz B, Bobo L, Mkocha $\mathrm{H}$, Lynch $\mathrm{M}$, Hsieh $\mathrm{YH}$, West S. Incidence of trichiasis in a cohort of women with and without scarring. Int J Epidemiol. 1999;28:1167-1171.

12. Schachter J, West SK, Mabey D, et al. Azithromycin in control of trachoma. Lancet. 1999;354:630-635.

13. Solomon AW, Holland MJ, Burton MJ, et al. Strategies for control of trachoma: observational study with quantitative PCR. Lancet. 2003;362:198-204.

14. Congdon N, West S, Vitale S, Katala S, Mmbaga BB. Exposure to children and risk of active trachoma in Tanzanian women. Am J Epidemiol. 1993;137:366-372.

15. Turner VM, West SK, Munoz B, et al. Risk factors for trichiasis in women in Kongwa, Tanzania: a case-control study. Int J Epidemiol. 1993;22:341-347.

16. Taylor H, Siler J, Mkocha H, Munoz B, West S. The natural history of endemic trachoma: a longitudinal study. Am J Trop Med Hyg. 1992;46:552-559.

17. Bailey R, Osmond C, Mabey D, Whittle HC, Ward ME. Analysis of the household distribution of trachoma in a Gambian village using a Monte Carlo simulation procedure. Int J Epidemiol. 1989;18: 944-951.

18. Katz J, Zeger S, Tielsch J. Village and household clustering of xerophthalmia and trachoma. Int J Epidemiol. 2001;17:865-869.

19. Alemayehu W, Melese M, Bejiga A, Worku A, Kebede W, Fantaye D. Surgery for trichiasis by ophthalmologists versus integrated eye care workers: a randomized trial. Opbthalmology. 2004;111:578584 .

20. Zhang H, Kandel R, Sharma B, Dean D. Risk factors for recurrence of postoperative trichiasis. Arch Ophthalmol. 2004;122:511-516.

21. West S, Munoz B, Lynch M, Kayongoya A, Mmbaga BB, Taylor H. Risk factors for constant severe trachoma among pre-school children in Kongwa, Tanzania. Am J Epidemiol. 1996;143:73-78. 